\title{
Photometric study of the eclipsing binary V1034 Sco
}

\author{
M. Y. Bouzid ${ }^{1}$, C. Sterken ${ }^{1, \star \star}$, and T. Pribulla ${ }^{2}$ \\ 1 Astronomy Group, Vrije Universiteit Brussels (VUB), Pleinlaan 2, 1050 Brussels, Belgium \\ e-mail: [ybouzid;csterken]@vub.ac.be \\ 2 Astronomical Institute of the Slovak Academy of Sciences, 05960 Tatranská Lomnica, The Slovak Republic \\ e-mail: pribulla@ta3.sk
}

Received 29 November 2004 / Accepted 14 March 2005

\begin{abstract}
We present multi-colour Strömgren photometry of the eclipsing binary V1034 Sco in the galactic open cluster NGC 6231, providing for the first time a full coverage of the orbital period ( $P=2.44$ days) and a complete analysis of the orbit. This extensive new photometry was analyzed together with multiband spectroscopy and results in an improved ephemeris, photometric elements and absolute parameters of the components. The system is close, but clearly detached. The masses of the components $M_{1}=16.8 \pm 0.5 M_{\odot}$ and $M_{2}=9.4 \pm 0.3 M_{\odot}$ do not correspond to the published spectral classification (O9III + B1III). The parameters of the components are consistent with the main-sequence luminosity class. We independently derive a distance modulus $V_{0}-M_{V}=10.73 \pm 0.02$.
\end{abstract}

Key words. instrumentation: photometers - binaries: eclipsing - methods: data analysis - methods: observational techniques: photometric - Hertzspring-Russell and C-M diagrams

\section{Introduction}

The study of eclipsing binary stars in open clusters is of fundamental importance since it leads to the determination of masses, luminosities and radii of the components. These parameters are crucial for the understanding of star-formation processes and of stellar evolution in general. NGC 6231 is a young southern cluster in the core of the Sco OB 1 association harboring several variable stars. According to Balona \& Laney (1995) the distance modulus of the cluster is $V_{0}-M_{V}=11.08$, interstellar reddening $E(b-y)=0.325$ and its approximate age is about $5 \mathrm{My}$.

V1034 Sco (SBL505, Seggewiss 224, SAAO 100, CPD $\left.-41^{\circ} 7742, V_{\max }=8.32\right)$ is one of the two known eclipsing binaries in NGC 6231 (the second being HD 152248). The system has drawn much attention since the first description of Struve (1944). The first determination of the orbital period was based on the spectroscopic observations by Hill et al. (1974) who found $P=2.446 \pm 0.005$ days. These authors reported a slightly eccentric $(e=0.07 \pm 0.04)$ orbit and detected only spectral lines of the primary component with semi-amplitude $K_{1}=162.5 \pm 8.2 \mathrm{~km} \mathrm{~s}^{-1}$. The photometric variability was found to be about 0.45 mag. Garcia \& Mermilliod (2001), on the basis of spectroscopic observations, found an orbital period $P=2.453087 \pm 0.000012$ days. Sana et al. (2003), using radial velocities of the primary component from the literature, found a period $P=2.44062 \pm 0.00005$ days and

* Based on observations obtained at ESO.

$\star \star$ Research Director, Belgian Fund for Scientific Research (FWO).

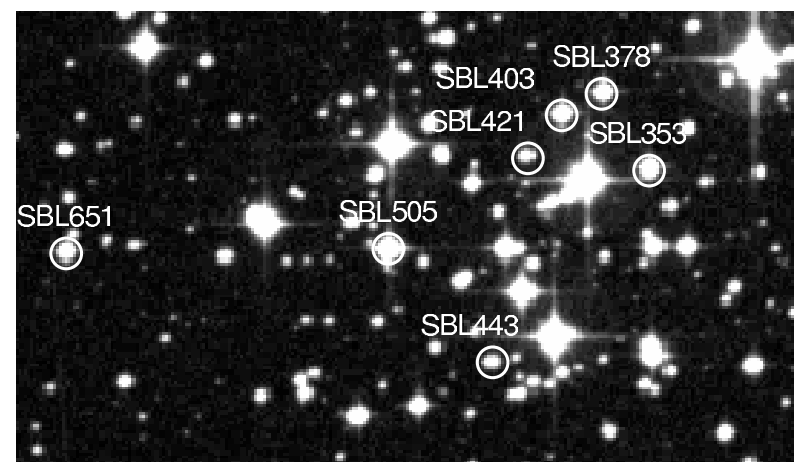

Fig. 1. Digital Sky Survey image of the center of NGC $6231\left(\alpha_{2000}=\right.$ $\left.16^{\mathrm{h}} 54^{\mathrm{m}} 8.5, \delta_{2000}=-41^{\circ} 49^{\prime} 36^{\prime \prime}\right)$, size $7.22 \times 4.23$ V1034 Sco (SBL505); The comparison stars are indicated.

spectroscopic elements $K_{1}=167.1 \pm 0.9 \mathrm{~km} \mathrm{~s}^{-1}, K_{2}=301.3 \pm$ $1.8 \mathrm{~km} \mathrm{~s}^{-1}, e=0.027 \pm 0.006, M_{1} \sin ^{3} i=16.69 \pm 0.25 M_{\odot}$ and $M_{2} \sin ^{3} i=9.25 \pm 0.12 M_{\odot}$. The components were classified as 09 III+B1 III. Since there were almost no photometric data available, precise masses and radii of the components remained unknown.

\section{New CCD observations}

uvby data were obtained at ESO in the framework of the "Long-term Photometry of Variables" (LTPV) project (Sterken $1983,1994)$ using the Danish 1.54-m telescope with a LORAL $2048 \times 2048$ CCD chip for the observations of 2000 June/July, 
and an EEV/MAT $2048 \times 4096$ chip since 2001 March. The observations were carried out in 2000 June/July, 2001 March, 2001 April and 2001 July. More than 3000 data points were obtained. The preliminary reduction of the frames (dark subtraction and flatfield correction) was performed in the usual way.

\section{Data reduction}

The photometric reduction was carried out with MOMF (Kjeldsen \& Frandsen 1992). This package is designed for CCD time-series photometry and uses a combination of PSF and aperture photometry.

In a first step we detected all stars with reasonable $\mathrm{S} / \mathrm{N}$ on a reference frame obtained under good sky conditions. We then determined the instrumental magnitudes of all detected stars in a non-differential way, and selected 6 constant stars (relatively bright stars not affected by bad columns and sufficiently distant from the edge in each frame). The weighted average of their magnitudes serves as an artificial constant reference magnitude for a given frame. The relative weights (proportional to $1 / \sigma^{3}$ ) for these stars were computed depending on the standard deviation determined on the longest and best observing run. The weights were kept the same for all nights. Because the precision of the aperture photometry depends on the aperture size, we selected the optimal aperture taking as a condition the lowest standard deviation for each star. The differential magnitudes of all measured stars were then obtained with respect to the artificial constant star in the same aperture. We also verified whether the lowest standard deviation did not occur by chance by plotting $\sigma$ versus aperture and magnitude versus aperture. From this, we determined that the best aperture was $3.4 \times F W H M$ in $u, v$ and $b$, and $4.2 \times F W H M$ in $y$.

\section{Orbital period}

Although Sana et al. (2003) determined the orbital period relatively precisely, the instant of spectroscopic conjunction remains rather uncertain. Hence we tried to improve the ephemeris of the system from our new photometry using the code TRIGON written by one of us (TP). The code uses trigonometric polynomial fitting to find optimal periodicities in the time series. To check the reliability of the solution we performed the period search in each photometric band separately. This resulted in approximate orbital periods in the range 2.44062-2.44065 days for all passbands. Then the time of the minimum light and orbital period were optimized using symmetric trigonometric polynomials:

$I=a_{0}+\sum_{n=1}^{N} a_{n} \cos \frac{2 \pi n\left(t-T_{0}\right)}{P}$,

where $t$ is the time of the observation, $I$ is the observed magnitude, $T_{0}$ is the minimum light and $P$ the orbital period. The non-linear optimization process converged for all Strömgren passbands. The best ephemeris in $u, v, b, y$ is:

MinI $=2451821.4361(4)+2.440629(5) \times E(u)$
MinI $=2451870.2500(3)+2.440658(4) \times E(v)$
MinI $=2451933.7069(3)+2.440656(5) \times E(b)$
MinI $=2451931.2652(2)+2.440646(4) \times E(y)$.
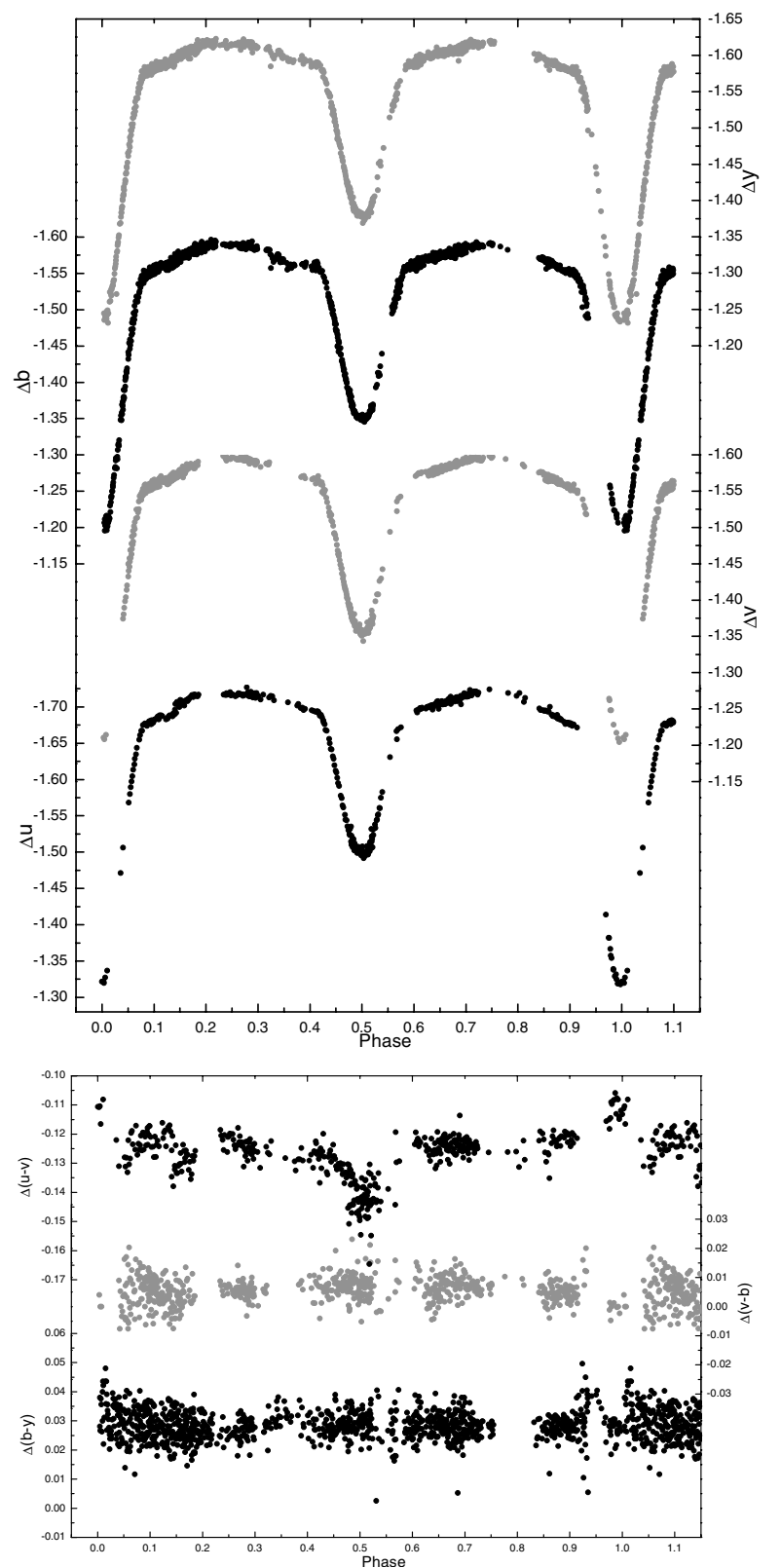

Fig. 2. Stromgren $u v b y$ light curves (top) and $b-y, v-b$ and $u-v$ color indices (bottom) folded with ephemeris (2) for the $y$ passband.

The ephemeris (2) in $y$, was used to phase all data (see Fig. 2). The uvby light curves show eclipses with depth 0.45 and $0.25 \mathrm{mag}$. The eclipses are partial - that is, there is no interval of constant light and the orbital inclination is relatively low.

\section{Modelling of light and radial velocity curves}

Our photometry of June 26, 2000-July 11, 2001 partly overlaps with the spectroscopic observations of Sana et al. (2003) which were obtained between July 1998 and May 2002. Hence we tried to perform simultaneous optimization of both datasets. The average radial velocities determined from several He I lines given in Table 1 of Sana et al. (2003) were used and 
the effective wavelength of the radial velocities was adopted to be $4400 \AA$, close to the average wavelength of the dataset.

The modelling of the photometric and spectroscopic observations was performed using the code ROCHE (Pribulla 2004) based on the principles of Wilson \& Devinney (1971) and Wilson (1979). The code assumes that the shapes of the components are given by the Roche equipotentials. The local monochromatic fluxes were interpolated from the ATLAS stellar atmosphere models (see Lejeune et al. 1997). Since V1034 Sco is a member of a young stellar cluster, we selected the models for rather high metallicity $Z=0.02$.

Monochromatic and bolometric limb darkening coefficients were interpolated after each optimization step from the extensive tables of Van Hamme (1993) according to the mean local gravity and temperature of both components.

We assumed a detached configuration, synchronous rotation of the components, a logarithmic limb darkening law and the temperature of the primary (more massive and hotter) component corresponding to its spectral type $T=33200 \mathrm{~K}$ (using the calibration of Popper 1980). Since both components are early-type stars we assumed radiative energy transfer in their envelopes and used gravity darkening coefficients $\beta_{1}=\beta_{2}=$ 0.25 and bolometric albedos $A_{1}=A_{2}=1.00$.

The curved maxima indicate that the system is rather close with fairly deformed components. This is in contradiction to the eccentricity detected from the spectroscopic data (see Sana et al. 2003; and Sect. 4 for the discussion). Hence, we assumed synchronous rotation and a circular orbit. All observables (light and radial velocity curves) were not phased a priori but left in heliocentric Julian time to optimize the ephemeris determination. The scatter of radial velocities was found by fitting the data under the assumption of the mass-point approximation. The resulting standard errors $\sigma_{\mathrm{RV} 1}=6.6 \mathrm{~km} \mathrm{~s}^{-1}$ and $\sigma_{\mathrm{RV} 2}=12.3 \mathrm{~km} \mathrm{~s}^{-1}$ were used to weight both radial-velocity datasets. The light curves were weighted according to the mean scatter in a given bandpass as determined by the trigonometric polynomial fitting.

The radial velocities of the secondary component, as determined by Sana et al. (2003), do not show any traces of the Rossiter-McLoughlin effect during its secondary eclipse (see Fig. 3). This can be for two reasons:

1 the fitting of the profiles of the secondary component during the eclipse by a Gaussian function is not appropriate due to the strong asymmetries of the profile and because of severe blending by the lines of the primary component;

2 the rotation of the secondary is asynchronous so that it does not rotate with respect to the inertial coordinate system.

Improper determination of the radial velocities of the secondary in the former possibility can be avoided by direct fitting of the line profiles with a binary model taking into account tidal, reflection and eclipse effects. We omitted 4 radial-velocity values during the secondary minimum at HJD $2400000+51302.847,51668.928,52039.783$, 52039.907 since they deviated from the solution because of line blending (Sana, private communication). Using the sum of reduced $\chi^{2}$ for LC and RV curves separately did not help to stabilize the solution. In this case the fit of LCs deteriorated
Table 1. The spectroscopic elements of V1034 Sco assuming photometric orbital period $P=2.440656$ days and circular or eccentric orbits. Spectroscopic elements: time of spectroscopic conjunction $T_{0}$; eccentricity $e$; longitude of periastron $\omega$; systemic velocity $V_{0}$; $\mathrm{RV}$ semi-amplitudes $K_{1}, K_{2}$. The time of the conjunction is given as $2451738+$. The parameters not adjusted but fixed in the solution are denoted by the superscript $f$.

\begin{tabular}{lllrr}
\hline \hline & \multicolumn{2}{c}{ Circular } & \multicolumn{2}{c}{ Eccentric } \\
Parameter & \multicolumn{2}{c}{$\sigma$} & & $\sigma$ \\
\hline$T_{0}[\mathrm{HJD}]$ & 0.4651 & 0.0034 & 0.4771 & 0.0054 \\
$e$ & $0.0^{f}$ & - & $0.026^{f}$ & 0.008 \\
$\omega[\mathrm{deg}]$ & - & - & 132 & 18 \\
$V_{0}\left[\mathrm{~km} \mathrm{~s}^{-1}\right]$ & +0.36 & 1.3 & +0.27 & 1.2 \\
$K_{1}\left[\mathrm{~km} \mathrm{~s}^{-1}\right]$ & 167.1 & 2.1 & 167.8 & 2.0 \\
$K_{2}\left[\mathrm{~km} \mathrm{~s}^{-1}\right]$ & 299.9 & 3.8 & 301.1 & 3.6 \\
\hline$\chi^{2}$ & 56.75 & - & 46.6 & - \\
\hline
\end{tabular}

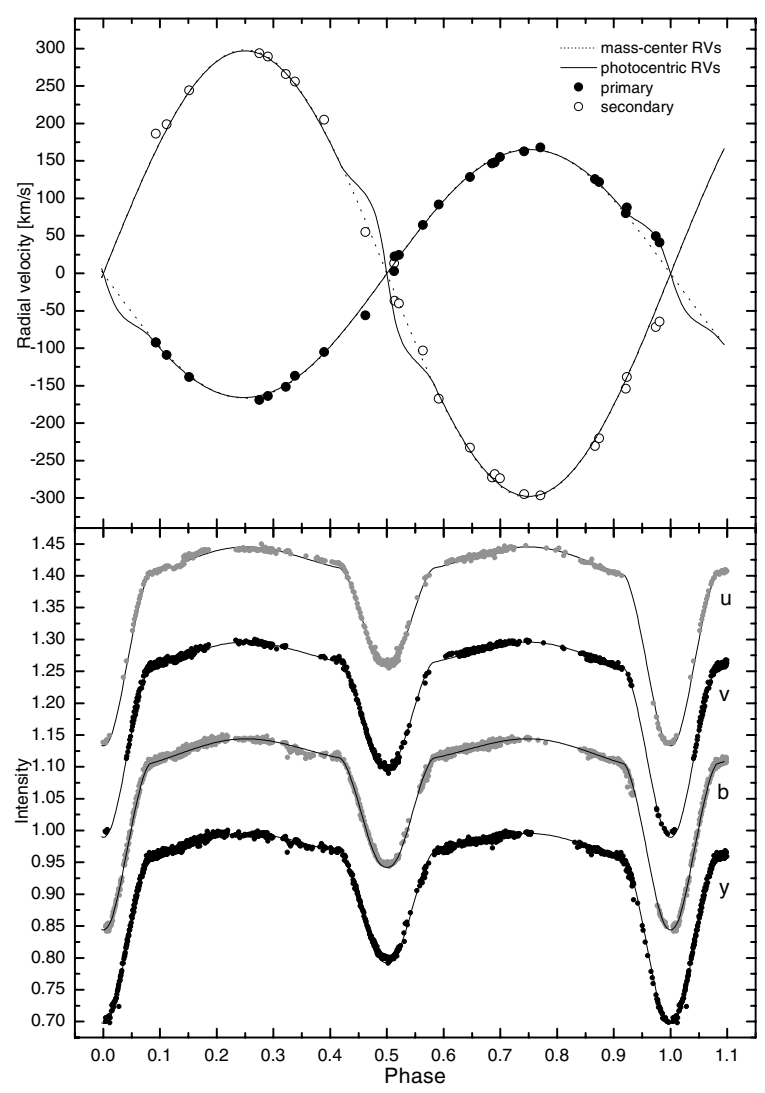

Fig. 3. The best fits to the Strömgren $u v b y$ light curves and radial velocities. The data are phased using the optimal ephemeris (Table 2).

significantly. Hence we first determined the spectroscopic elements from radial velocities. These were computed under the assumption of the photometric orbital period $(P=$ 2.440656 days) and a circular and eccentric orbit. Due to the different precision of the primary and secondary component datasets, the relative weight $w_{\mathrm{sec}} / w_{\mathrm{pri}}=\sigma_{\mathrm{pri}}^{2} / \sigma_{\mathrm{sec}}^{2}=0.303$ was used. The resulting elements are given in Table 1. 
Table 2. The combined photometric and spectroscopic solution of V1034 Sco. Photometric elements: inclination angle $i$; mass ratio $q=$ $m_{2} / m_{1}$; surface equipotentials $\omega_{1,2}$; mean volume radii of the components $r_{1,2}$; polar temperatures of the components $T_{1,2}$; systemic velocity $V_{0}$; sum of the semi-amplitudes of the radial-velocity changes $K_{1,2}$; time of the conjunction (Julian date is given 2451738) $J D_{0}$; orbital period $P$. The parameters not adjusted but fixed in the solution are denoted by the superscript $f$. The $\chi^{2}$ are normalised.

\begin{tabular}{lllrr}
\hline \hline & \multicolumn{2}{c}{ O9V } & \multicolumn{2}{c}{$\mathrm{B} 1 \mathrm{~V}$} \\
Parameter & & $\sigma$ & 81.5 & 0.09 \\
\hline$i$ [deg] & 81.4 & 0.10 & $0.5572^{f}$ & - \\
$q$ & $0.5572^{f}$ & - & 3.663 & 0.003 \\
$\Omega_{1}$ & 3.657 & 0.003 & 4.270 & 0.008 \\
$\Omega_{2}$ & 4.256 & 0.006 & 0.3283 & 0.0005 \\
$r_{1}$ & 0.3289 & 0.0004 & 0.1841 & 0.0007 \\
$r_{2}$ & 0.1842 & 0.0006 & $26200^{f}$ & - \\
$T_{1}[\mathrm{~K}]$ & $33200^{f}$ & - & 20350 & 25 \\
$T_{2}[\mathrm{~K}]$ & 26330 & 30 & -0.4 & 1.4 \\
$V_{0}\left[\mathrm{~km} \mathrm{~s}{ }^{-1}\right]$ & -0.3 & 1.3 & 464.6 & 4.5 \\
$K_{1,2}\left[\mathrm{~km} \mathrm{~s}^{-1}\right]$ & 464.6 & 4.6 & 0.4536 & 0.0003 \\
\hline$J D_{0}$ & 0.4533 & 0.0002 & 1.415 & - \\
$P[\mathrm{days}]$ & 2.440656 & 0.000002 & 2.440656 & 0.000002 \\
\hline$\chi_{\mathrm{LC}}^{2}$ & 1.361 & - & 1.066 & - \\
$\chi_{\mathrm{RV}}^{2}$ & 1.092 & - & 1.405 & - \\
$\chi^{2}$ & 1.353 & - & & \\
\hline
\end{tabular}

Although the slightly eccentric orbit gives smaller $\chi^{2}$, the light curves are perfectly symmetric (see Sect. 6), and therefore, we assume a circular orbit in the photometric solution, fixing the mass ratio $q$ from the spectroscopy.

The resulting best fits of all light and radial velocity data are shown in Fig. 3. The light curves are quite well explained by the fits, except the minima where the observed depth is not perfectly fitted in all passbands. This is partially due to using uvby data in the instrumental system since the computed fits depend on the adopted effective wavelengths. Corresponding parameters of the solution together with standard errors are listed in Table 2.

\section{Orbital eccentricity}

As pointed out by Sana et al. (2003), the radial velocities of the system suggest a low eccentricity of about 0.03 . It is interesting to note that the orientation of the orbit is quite different for three solutions in their Table 4, making the given small eccentricity rather improbable.

If there really is a small but definite eccentricity, we can expect a small shift in the secondary minimum with respect to phase 0.5 . The theoretical shift of the secondary minima is (Kallrath \& Milone 1999):

$\Delta T=\operatorname{Min} I I-\operatorname{Min} I-\frac{P}{2}=\frac{2 P e \cos \omega}{\pi}\left(1+\frac{1}{\sin ^{2} i}\right)$.
Using three orbital solutions of Sana et al. (2003) from their Table 4 and the inclination angle $i=81.5$ from our combined solution, we obtain phase shifts $-0.022,-0.015$ and +0.017 . The observed phase of the secondary minima in our $u v b y$ data, determined using Kwee \& van Woerden's (1956) method is $0.5002 \pm 0.0006$.

The orbital eccentricity can be assessed using the circularization time scales of Claret et al. (1995). The circularization time-scale of a star in a close binary is:

$\tau_{\text {circ }}^{1,2}=9.4 \times 10^{3+\gamma-N / 4}(1+q)^{2 / 3} \beta^{-2} L^{-1 / 4} M^{23 / 12} R^{-5} P^{49 / 12}$,

where $L, M, R$ are stellar luminosity, mass and radius, $q$ is the mass ratio, $\beta$ is the gyration radius, $P$ is the orbital period, $N$ depends on the mode of energy transfer $(N=0.0$ for radiative envelopes) and $\gamma \approx 1$. The circularization time scale for the system is computed as:

$\frac{1}{\tau_{\text {circ }}}=\frac{1}{\tau_{\text {circ }}^{1}}+\frac{1}{\tau_{\text {circ }}^{2}}$,

where $\tau_{\text {circ }}^{1}$ and $\tau_{\text {circ }}^{2}$ are the circularization time scales for the components (Eq. (5)). Using absolute parameters of the system from Table 2 (for $T_{1}=33200 \mathrm{~K}$ ), and gyration radii for the components from Claret (1995), we obtain the circularization time scale for V1034 Sco as $\tau_{\text {circ }} \approx 40000$ years. Hence, we regard the small eccentricity found in the spectroscopy as a possible result of circumstellar matter or other radiating material not connected to the orbital motion of the binary.

\section{Absolute parameters and evolutionary status}

The absolute parameters of the component stars derived from the combined photometric and spectroscopic solution are given in Table 3. All errors are derived from standard errors of independent parameters from the combined solution. The error on the luminosity of the primary is here dependent on the error of its radius only. As already noticed by Sana et al. (2003), the luminosity class of both components is very probably $\mathrm{V}$, and not III or IV as inferred by spectroscopy. The mass and radius of both components (Table 3 ) is small, even for MS stars of accepted spectral types. The discrepancy between the determined masses and radii can be inferred using the evolutionary tracks from Claret (1995). As seen in Fig. 4, both stars are highly overluminous for their mass. We, therefore, determined four additional combined solutions of light and radial velocity observations assuming later spectral types of the primary component $(\mathrm{O} 9 \mathrm{~V}, 09.5 \mathrm{~V}, \mathrm{~B} 0 \mathrm{~V}, \mathrm{~B} 0.5 \mathrm{~V}$ and B1V). The parameters for the marginal solution are given in Tables 2 and 3. We found that the solution intersects the isochrone of $5 \mathrm{Myr}$ (the age derived by Balona \& Laney 1995) for the B0V + B2V classification and which is located in the region of slightly more massive stars $\left(M_{1} \approx 19 M_{\odot}\right.$ and $\left.M_{2} \approx 11 M_{\odot}\right)$. While the masses of the components as determined from the combined solution $\left(M_{1} \approx 16.8 M_{\odot}\right.$ and $\left.M_{2} \approx 9.4 M_{\odot}\right)$ agree with those predicted by stellar models for B0.5V + B2V classification, the intersection here occurs at about 6-7 My (Fig. 4) which represents the best agreement between our results and the stellar models.

The distance to the cluster is known fairly well. Balona \& Laney (1995), assuming that most stars of the cluster are 
Table 3. Absolute parameters of V1034 Sco: semi-major axis $a$; mass $M$, radius $R$, luminosity $L$; mean surface gravity $g$; mean density $\rho$.

\begin{tabular}{lrrrr}
\hline \hline & \multicolumn{2}{c}{ O9V } & \multicolumn{2}{c}{$\mathrm{B} 1 \mathrm{~V}$} \\
Parameter & & \multicolumn{1}{c}{$\sigma$} & & $\sigma$ \\
\hline$a\left[R_{\odot}\right]$ & 22.65 & 0.21 & 22.66 & 0.22 \\
Primary component & & & \\
$M\left[M_{\odot}\right]$ & 16.83 & 0.48 & 16.84 & 0.49 \\
$R\left[R_{\odot}\right]$ & 7.45 & 0.07 & 7.44 & 0.08 \\
$L\left[L_{\odot}\right]$ & 60950 & 1150 & 23600 & 510 \\
$\log g[\mathrm{cg}]$ & 3.90 & - & 3.90 & - \\
$\log \left(\rho / \rho_{\odot}\right)$ & -1.389 & - & -1.389 & - \\
Secondary component & & & \\
$M\left[M_{\odot}\right]$ & 9.38 & 0.27 & 9.38 & 0.27 \\
$R\left[R_{\odot}\right]$ & 4.18 & 0.04 & 4.17 & 0.04 \\
$L\left[L_{\odot}\right]$ & 7920 & 160 & 2830 & 60 \\
$\log g[\mathrm{cgs}]$ & 4.16 & - & 4.16 & - \\
$\log \left(\rho / \rho_{\odot}\right)$ & -0.636 & - & -0.636 & - \\
\hline
\end{tabular}

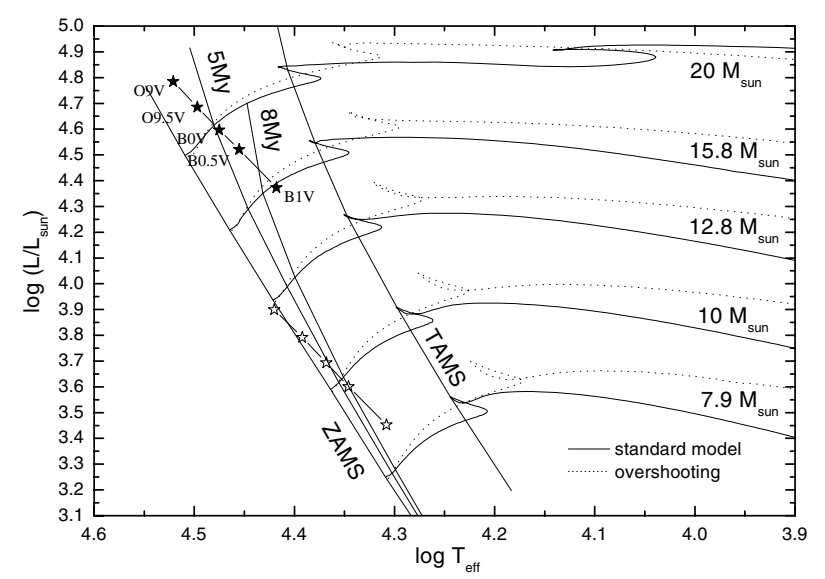

Fig. 4. The evolutionary tracks for massive stars with $7.9 M_{\odot}<M<$ $20 M_{\odot}, Z=0.02$ and initial hydrogen content $X=0.80$. The standard models are plotted in solid lines, the models taking into account the overshooting are repreented by dotted lines (overshooting parameter $d=0.20$ ). The positions of the components for several classifications of the primary are marked by asterisks.

on the ZAMS, determined the distance modulus $\left(V_{0}-M_{V}\right)=$ $11.08 \pm 0.05$ equivalent to the distance $1644 \pm 38$ pc. The distance modulus determined from the revised Hipparcos data on 6 member stars is, however, only $8.9 \pm 0.5$ (Makarov 2003).

The distance to V1034 Sco can be determined independently using the new combined solution. Taking first the parameters for the original solution with $T_{1}=33200 \mathrm{~K}$ (corresponding to $\mathrm{O} 9 \mathrm{~V}$ ) and using the bolometric corrections from the calibration of Popper (1980) and $M_{V \odot}=4.83$, we get $M_{V 1}=-3.93 \pm 0.02$ and $M_{V 2}=-2.33 \pm 0.02$ resulting in a combined absolute magnitude $M_{V}=-4.16 \pm 0.02$. The observed maximum brightness (out of eclipse) of the system is $V=8.319$ (Balona \& Laney 1995). Using the mean interstellar absorption towards the cluster $A_{V}=1.423$ (Balona \& Laney 1995), we get a distance modulus $V_{0}-M_{V}=11.05 \pm 0.02$, equivalent to a distance of $1.62 \mathrm{kpc}$. Taking parameters from the alternative solution with $T_{1}=26200 \mathrm{~K}$ (corresponding to a B1V primary), we get the combined maximum absolute magnitude of the binary as $M_{V}=-3.83$ leading to a distance modulus $10.60 \pm 0.01$ equivalent to a distance of $1.32 \mathrm{kpc}$. The best agreement of the determined masses with those predicted by stellar models occurs for a B0.5V primary as mentioned, whith $\left(V_{0}-M_{V}\right)=10.73$ yielding a somewhat smaller distance to the cluster than previously determined.

\section{Discussion and conclusions}

Simultaneous analysis of published radial velocity data of Sana et al. (2003) and high-precision multi-color photometry of the eclipsing binary V1034 Sco in the open southern cluster NGC 6231 leads to orbital elements and absolute parameters of the components. The orientation of the orbital plane of the system is far from edge-on, the orbital inclination being $\approx 81^{\circ}$. The combination of the spectroscopic elements and the inclination angle gives the following masses of the components; $M_{1}=16.8 \pm 0.5 M_{\odot}$ and $M_{2}=9.4 \pm 0.3 M_{\odot}$.

The system is clearly detached with components filling just $44 \%$ and $17 \%$ of their Roche lobes. Hence, it is very probable that the system is before any mass transfer and that both components evolve independently. This can well be documented in the H-R diagram (Fig. 4), where the components are on the same isochrones. Both components are still on the mainsequence and relatively far from the turn-off point of the cluster. The primary, more massive component will reach its Roche lobe (with volume mean radius $9.8 R_{\odot}$ ) in approximately $6 \mathrm{My}$ when the system enters a semi-detached phase. A good agreement of observed masses, temperatures and luminosities in the theoretical H-R diagram (evolutionary tracks of Claret 1995) can be attained for a somewhat later spectral classification than previously published.

Acknowledgements. This work has been supported by "IAP P5/36" Interuniversity Attraction Poles Programme of the Belgian Federal Office for Scientific, Technical and Cultural Affairs, the Belgian Fund for Scientific Research (FWO), the Flemish Ministry for Foreign Policy, European Affairs, Science and Technology, under contract BIL 01/3 and by the Danish Natural Science Research Council through the centre for Ground-Based Observational Astronomy. The stay of TP at VUB was partly supported by the Science and Technology Assistance Agency under the contract No. APVT-20-014402.

\section{References}

Arentoft, T., Sterken, C., Knudsen, M. R., et al. 2001, A\&A, 380, 599 Garcia, B., \& Mermilliod, J. C. 2001, A\&AS, 122, 136

Balona, L. A., \& Laney, C. D. 1995, MNRAS, 276, 627

Balona, L. A., \& Engelbrecht, C. A. 1985, MNRAS, 212, 889

Balona, L. A., \& Shobbrook, R. R. 1984, MNRAS, 211, 375

Balona, L. A. 1983, MNRAS, 203, 1041

Baume, G., Vázquez, R. A., \& Feinstein, A. 1999, A\&AS, 137, 233

Claret, A. 1995, A\&AS, 109, 441 
Claret, A., Gimenez, A., \& Cunha, N. C. S. 1995, A\&AS, 299, 724

Garcia, B., \& Mermilliod, J. C. 2001, A\&A, 368, 122

Hill, G., Crawford, D. L., \& Barnes, J. V. 1974, AJ, 79, 1271

Kallrath, J., \& Milone, E. F. 1999, Eclipsing Binary Stars (New York: Springer Verlag)

Kwee, K. K., \& van Woerden, H. 1956, Bull. Astron. Inst. Nether., 12, 327

Popper, D. M. 1980, ARA\&A, 18, 115

Pribulla, T. 2004, in Spectroscopically and Spatially Resolving the Components of the Close Binary Stars, ed. R. W. Hilditch et al., ASP Conf. Ser., 318, 117

Sana, H., Hensberge, H., \& Rauw, G. 2003, A\&A, 1063, 1074

Sterken, C. 1983, The ESO Messenger, 33, 10
Sterken, C. 1994, in The Impact of Long-Term Monitoring on Variable-Star Research, NATO ARW, ed. C. Sterken, \& M. de Groot, NATO ASI Series C, 436, 1 (Kluwer Ac. Publ.) Struve, O. 1944, ApJ, 100, 189

Sung, H., Bessell, M. S., \& Lee, S.-W. 1998, AJ, 115, 734 (SBL)

Kjeldsen, H., \& Frandsen, S. 1992, PASP, 104, 413

Lejeune, T., Cuisinier, F., \& Buser, R. 1997, A\&AS, 125, 229

Makarov, V. V. 2003, AJ, 126, 2408

van Genderen, A. M., Bijleveld, W., \& van Groningen, E. 1984, A\&A, 58,537

Van Hamme, W. 1993, AJ, 106, 2096

Wilson, R. E. 1979, ApJ, 234, 1054

Wilson, R. E., \& Devinney, E. J. 1971, ApJ, 166, 605 